\title{
Intramolecular photoelectron diffraction in the gas phase
}

\author{
K. Ueda, ${ }^{1, a)}$ C. Miron, ${ }^{2}$ E. Plésiat, ${ }^{3}$ L. Argenti, ${ }^{3}$ M. Patanen, ${ }^{2}$ K. Kooser, ${ }^{4}$ D. Ayuso, ${ }^{3}$ \\ S. Mondal, ${ }^{1}$ M. Kimura, ${ }^{1}$ K. Sakai, ${ }^{1}$ O. Travnikova, ${ }^{2}$ A. Palacios, ${ }^{3}$ P. Decleva,${ }^{5}$ E. Kukk, ${ }^{4}$ \\ and F. Martín ${ }^{3,6, b)}$ \\ ${ }^{1}$ Institute of Multidisciplinary Research for Advanced Materials, Tohoku University, Sendai 980-8577, \\ Japan \\ ${ }^{2}$ Synchrotron SOLEIL, L'Orme des Merisiers, Saint-Aubin, BP 48, 91192 Gif-sur-Yvette Cedex, France \\ ${ }^{3}$ Departamento de Química, Módulo 13, Universidad Autónoma de Madrid, 28049 Madrid, Spain \\ ${ }^{4}$ Department of Physics and Astronomy, University of Turku, 20014 Turku, Finland \\ ${ }^{5}$ Dipartimento di Scienze Chimiche, Universita' di Trieste, 34127 Trieste, and CNR-IOM, Trieste, Italy \\ ${ }^{6}$ Instituto Madrileño de Estudios Avanzados en Nanociencia (IMDEA-Nanociencia), Cantoblanco, \\ 28049 Madrid, Spain
}

(Received 27 May 2013; accepted 5 August 2013; published online 27 September 2013)

\begin{abstract}
We report unambiguous experimental and theoretical evidence of intramolecular photoelectron diffraction in the collective vibrational excitation that accompanies high-energy photoionization of gas-phase $\mathrm{CF}_{4}, \mathrm{BF}_{3}$, and $\mathrm{CH}_{4}$ from the $1 s$ orbital of the central atom. We show that the ratios between vibrationally resolved photoionization cross sections ( $v$-ratios) exhibit pronounced oscillations as a function of photon energy, which is the fingerprint of electron diffraction by the surrounding atomic centers. This interpretation is supported by the excellent agreement between first-principles static-exchange and time-dependent density functional theory calculations and high resolution measurements, as well as by qualitative agreement at high energies with a model in which atomic displacements are treated to first order of perturbation theory. The latter model allows us to rationalize the results for all the $v$-ratios in terms of a generalized $v$-ratio, which contains information on the structure of the above three molecules and the corresponding molecular cations. A fit of the measured $v$-ratios to a simple formula based on this model suggests that the method could be used to obtain structural information of both neutral and ionic molecular species.

(C) 2013 AIP Publishing LLC. [http://dx.doi.org/10.1063/1.4820814]
\end{abstract}

\section{INTRODUCTION}

X-ray light efficiently ionizes the inner and valence shells of atoms and molecules, leading to short-wavelength electrons that can be scattered coherently (or diffracted) by the surrounding atomic constituents. Thus, scattered photoelectrons carry information about the structure of the system, which could in principle be extracted by fitting the measured photoelectron spectra to different analytical formulas. The NEXAFS (near-edge X-ray absorption fine structure) or EXAFS (extended X-ray absorption fine structure) techniques are based on this principle ${ }^{1,2}$ and are widely used to obtain structural information of crystals as well as bulk amorphous materials. ${ }^{3-9}$

Determining the structure of isolated molecules by photoelectron spectroscopy is more difficult due to the low density of the gas phase in comparison with that of bulk materials. Indeed, diffraction is clearly observed in the condensed phase because the relatively low intensity of the standard Xray sources used in the above mentioned techniques is amply compensated by the large number of atoms or molecules present in the sample (which is of the order of Avogadro's number, i.e., $\sim 10^{23}$ atoms or molecules per mole). In contrast,

\footnotetext{
a)Electronic mail: ueda@ tagen.tohoku.ac.jp

b) Electronic mail: fernando.martin@uam.es
}

for structural determination of isolated molecules one has to work with gases at very low pressures, i.e., densities as low as $10^{13}-10^{15}$ molecules per $\mathrm{cm}^{3}$. To overcome this difficulty, in addition to past and current developments in gas-phase EXAFS, ${ }^{10-12}$ alternative methods are being explored. One of these takes advantage of the high brightness of $\mathrm{x}$-ray free electron lasers, which should be ideal to obtain instantaneous and time-resolved pictures of molecular structure through the so-called photoelectron holography. ${ }^{13}$ A more sophisticated strategy, already put in practice, consists in combining intense infrared femtosecond lasers ${ }^{14-16}$ with laser-induced electron diffraction. In these experiments, the oscillating electric field of the laser forces a fraction of the tunneled electron to go back to the parent ion where it can diffract, thus conveying the necessary information to extract the structure of the molecule. Very recent experimental work has shown that this technique can provide sub- $\AA$ spatial resolution and sub-femtosecond temporal resolution. Another more traditional method consists in measuring molecular-frame photoelectron angular distributions (MFPAD) resulting from photoionization with synchrotron radiation. ${ }^{17,18}$ In particular, it has been shown that, in some cases, MFPADs associated with very slow electrons provide a direct imaging of molecular structure. ${ }^{19,20}$

A common denominator in the above examples is that photoelectron spectra must be recorded (at least) as a function of the electron ejection angle. This requires the use of rather 
sophisticated coincidence setups with high angular resolution and high detection efficiency, ${ }^{21,22}$ which are not always available or applicable. However, recent work on the photoionization of diatomic molecules by synchrotron radiation ${ }^{23,24}$ has shown that, under some circumstances, photoelectron spectra integrated over electron ejection angle might also be a valuable tool for structural determination. In particular, experiments and theoretical calculations performed on $\mathrm{H}_{2}, \mathrm{~N}_{2}$, and $\mathrm{CO}$ molecules have shown that the ratios of vibrationally resolved photoionization cross sections (hereafter called $v$ ratios) oscillate with photon energy and that this is due to the coherent electron emission from the two atomic centers. ${ }^{23}$ The frequency of the oscillations depends on the internuclear distance, hence they necessarily convey information about molecular structure. Oscillations resulting from coherent electron emission have also been observed in acetylene. ${ }^{25}$ There is recent evidence that electrons ejected from a very localized region of a molecule, such as the $1 s$ orbital of first-row atoms, also lead to oscillating patterns in the $v$-ratios as a result of electron scattering by the peripheral atomic centers of the molecule. ${ }^{26}$

In this paper, we unambiguously show that the $v$-ratios for photoionization of $\mathrm{CF}_{4}, \mathrm{BF}_{3}$, and $\mathrm{CH}_{4}$ from the $1 s$ orbital of the central atom strongly oscillate as functions of photoelectron energy. The very good agreement between first-principles static-exchange and time-dependent density functional theory calculations and high resolution measurements, as well as the qualitative agreement at high energies with a simple model show that the origin of these oscillations is the diffraction by the surrounding $\mathrm{H}$ and $\mathrm{F}$ atoms. A sketch of the phenomenon is shown in Fig. 1 for the case of the $\mathrm{BF}_{3}$

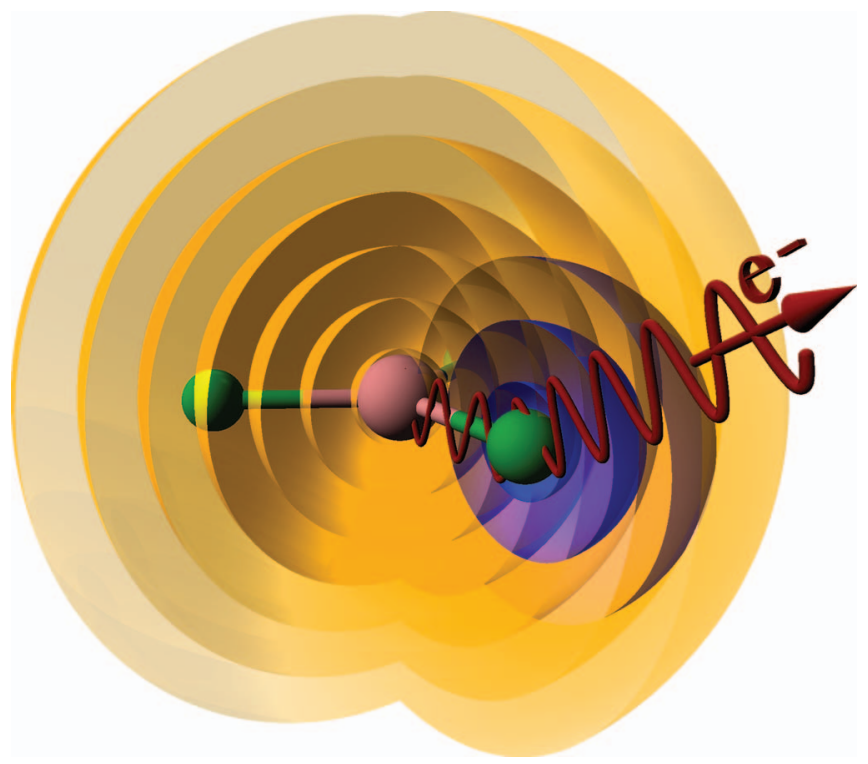

FIG. 1. Intramolecular electron diffraction in $\mathrm{BF}_{3}$. The $1 s$ electron of the central boron atom, which is localized around the boron nucleus, is ionized after absorption of an X-ray photon, leading, in a simplified picture to the yellow spherical wave. This wave is subsequently diffracted by the neighboring fluorine atoms, leading to secondary spherical waves as that plotted in blue around one of the fluorine atoms for an electron escaping along the direction represented by the red arrow. The combination of the yellow and blue waves leads to interferences that carry structural information about the parent neutral $\mathrm{BF}_{3}$ molecule and the final $\mathrm{BF}_{3}^{+}$molecular ion. molecule. From these results we have approximately determined the bond distances for the three molecules and their corresponding molecular cations. Thus, the present work provides an alternative and a complement to gas-phase electron diffraction (GED) techniques, ${ }^{27-30}$ which have been successfully used to retrieve three-dimensional structural information from molecules in the gas phase but would be difficult to apply to molecular cations.

\section{METHODS}

The experimental and theoretical methods have been explained in detail in previous work. ${ }^{26,31} \mathrm{~A}$ brief summary is presented below.

The experiment was performed at the PLEIADES beamline $^{32}(70-1000 \mathrm{eV})$ for ultrahigh resolution spectroscopic studies ${ }^{33,34}$ of isolated species at the $2.75 \mathrm{GeV}$ French national synchrotron radiation laboratory SOLEIL. Linearly polarized light with the polarization vector at $54.7^{\circ}$ with respect to the electron detection axis has been used with a spectral broadening of $55 \mathrm{meV}$ or less. The electron spectrometer resolution was better than $40 \mathrm{meV}$. The $1 \mathrm{~s}$ photoelectron spectra have been analyzed through a least-squares fitting procedure by using macro package SPANCF for Igor Pro. ${ }^{35}$

For a given molecule, all spectra were fitted simultaneously. Post-collisional interaction (PCI) line shapes ${ }^{36}$ were used in the fitting process with the Lorentzian width as a free parameter, but common for all the lines and spectra of the molecule. The Gaussian width representing the instrumental and translational Doppler broadenings was also kept as free parameter, but constrained to be the same to all peaks in the spectra. Rotational Doppler broadening ${ }^{37,38}$ is absent in the present case, because the electron emission takes place from the center of mass of the molecules. As the initial guesses were obtained from the fitted Ar calibration data, only small variations from the initial values were observed. The asymmetry leading to the low energy tail due to the PCI effect was calculated by using the formula given in Ref. 39. The energy spacing of the symmetric stretching vibrational sublevels was kept constant. In the data analysis, the recoilinduced excitations of asymmetric vibrations were taken into account by simultaneously fitting the asymmetric stretching and bending vibrational peaks with fixed energy separations for each mode, and their intensities were estimated following the method described in Ref. 40. In this way, individual vibrational progressions for the symmetric stretching, asymmetric bending, and asymmetric stretching modes could be obtained.

Vibrationally resolved photoionization cross sections were evaluated within the dipole and Born-Oppenheimer approximations. Bound and continuum electronic wave functions were calculated by using the static-exchange density functional theory (DFT) and the time-dependent density functional theory (TDDFT) as described in Refs. 26 and 31. In the latter works, the methods originally developed in, e.g., Refs. 41-44 to treat molecular photoionization in the fixednuclei approximation were extended to provide the electronic continuum wave functions and the corresponding potential energy surfaces over a wide range of nuclear positions. The electronic Kohn-Sham equations were solved by using a 
representation in a basis of B-spline functions. ${ }^{45}$ The vibrational wave function was written as a product of single-mode vibrational functions resulting from the solution of the vibrational Schrödinger equation in a basis of B-splines. As photoionization mainly leads to molecular cations with excitation in the symmetric stretching mode, calculations of the potential energy surfaces were restricted to this normal coordinate by keeping all the other normal coordinates frozen at their values of the equilibrium geometry. The method has been shown to provide accurate vibrationally resolved photoionization cross sections for $\mathrm{N}_{2}, \mathrm{CO}$, and $\mathrm{C}_{2} \mathrm{H}_{2} \cdot{ }^{23,25,31}$

\section{RESULTS}

As an illustration, Fig. 2 shows the photoelectron spectra of $\mathrm{BF}_{3}$ and $\mathrm{CF}_{4}$ at photon energies 518 and $383 \mathrm{eV}$, respectively. The figure also shows the results of the least-squares fit. As discussed in earlier work, ${ }^{40,46}$ the most prominent vibrational progression corresponds to the symmetric stretching mode. For this reason, all comparisons between experiment and theory reported below will be restricted to this mode. Recoil leads to a redistribution of the available energy
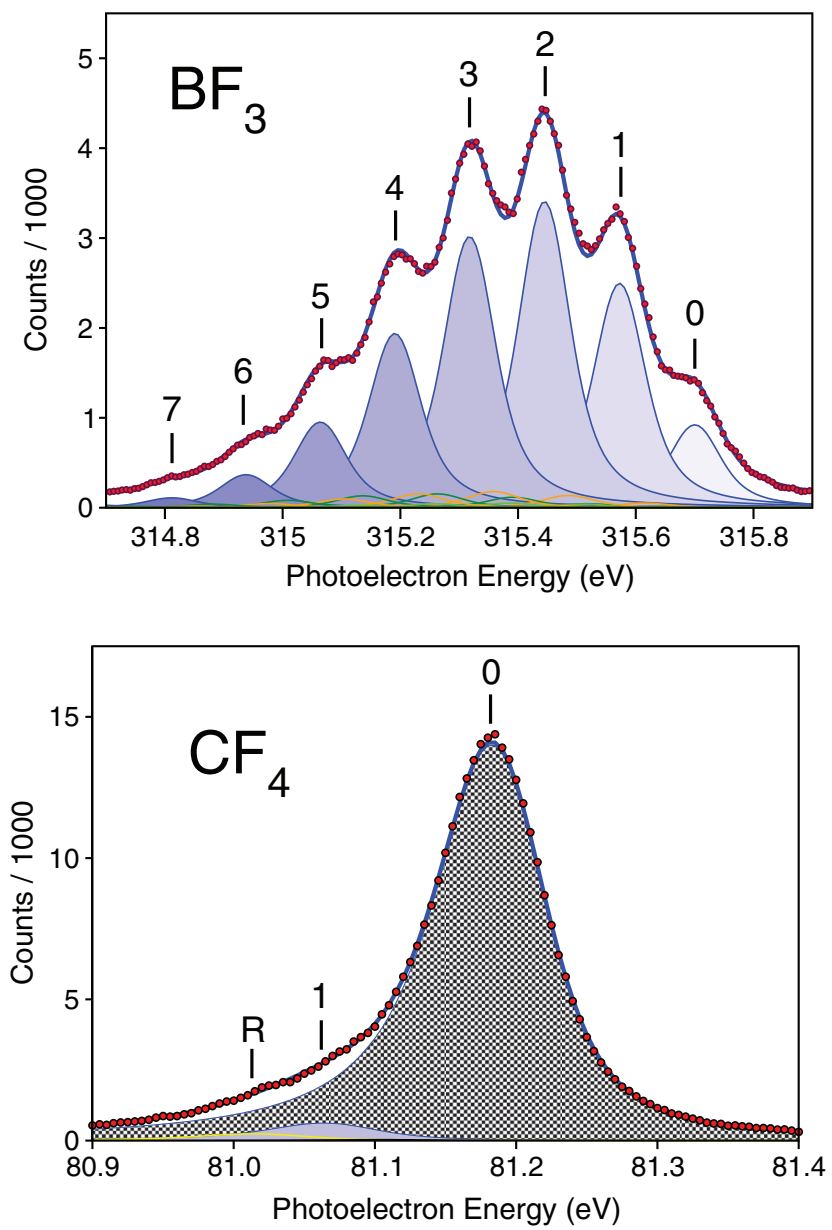

FIG. 2. Photoelectron spectra of $\mathrm{BF}_{3}$ (left) and $\mathrm{CF}_{4}$ (right) taken at $h v=518$ and $383 \mathrm{eV}$, respectively. Experimental results: circles. Thick blue line: fit of the experimental data. Thin blue lines enclosing shaded areas: vibrational progression associated with the symmetric stretching mode. Other thin lines: contribution of other modes resulting from recoil. Peak labels indicate the vibrational quantum number $v$ in the $\mathrm{BF}_{3}^{+}$symmetric stretching mode. even on symmetry-forbidden vibrational modes of the residual cation. For $\mathrm{CF}_{4}$, recoil leads to excitation of the asymmetric stretching and bending vibrations of the $\mathrm{CF}_{4}^{+}$ion, which superimpose to the $v=1$ symmetric stretching vibrational components. ${ }^{40}$ A similar effect has been observed for $\mathrm{CH}_{4}$ for the $v=1$ and $v=2$ components. ${ }^{47}$ Figure 2 shows that vibrational excitations due to recoil effects are much less pronounced for $\mathrm{BF}_{3}$ than for $\mathrm{CF}_{4}$ (and $\mathrm{CH}_{4}{ }^{47}$ ). As can be seen, $\mathrm{BF}_{3}$ photoionization leads to a large progression of vibrational levels in its symmetric stretching mode. This is the consequence of the favorable Franck-Condon (FC) overlap between the initial and several final vibrational wave functions, which results from the fact that the $\mathrm{B}-\mathrm{F}$ bond distances are significantly different in $\mathrm{BF}_{3}$ and $\mathrm{BF}_{3}^{+}$. For $\mathrm{CF}_{4}\left(\mathrm{CH}_{4}\right)$, the progression is limited to two (three) vibrational levels because the upper and the lower potential energy surfaces are very similar in the region close to the equilibrium geometry (in other words, the dominant FC overlap corresponds to the $v^{\prime}=0 \rightarrow v=0$ transition). For the three systems, the intensity of the measured peaks decreases very rapidly with photon energy. This rapid decrease usually washes out the oscillations arising from scattering effects as those sought for in this work. The problem is overcome in the $v$-ratios because the numerator and the denominator decrease with a similar rate. In spite of this, one cannot avoid long-time sample exposures due to the smallness of the cross sections at high photon energies.

Figure 3 shows the measured and calculated $v$-ratios as functions of photoelectron momentum for the symmetric stretching mode of $\mathrm{BF}_{3}$. The $v$-ratios exhibit pronounced oscillations superimposed to a nearly flat background. The agreement between theory and experiment is very good, even for the smallest $v$-ratios. In particular, the excellent agreement between the results from static-exchange DFT and the more elaborate TDDFT indicates that one can rely on the former to interpret the experimental findings. As can be seen in Fig. 3, the overall shape of the $v$-ratios is very similar for all $v \geq 3$; for $v=0$ and $v=1$, the oscillations are essentially identical but are perfectly out of phase from those with $v \geq 3$. For $\mathrm{CH}_{4}$, the $v=1 / v=0$ and $v=2 / v=0$ ratios reported in Ref. 26 are also very similar. For $\mathrm{CF}_{4}$, the present experiment can only provide the $v=1 / v=0$ ratio, but the various ones obtained from the static-exchange DFT and TDDFT calculations again resemble each other.

\section{DISCUSSION}

The above results suggest that, for a given molecule, all $v$-ratios carry the same structural information. As the amplitude of the oscillations is a small fraction of the vibrationally resolved cross section and, in particular, of the value that is obtained for this cross section within the FC approximation, one can assume that the variation of the transition dipole matrix element with the atomic displacements can be treated to first order in a Taylor expansion around the equilibrium geometry. Neglecting non-totally symmetric modes and assuming the validity of the harmonic approximation (which, as our calculations show, is a very good approximation for the low values of $v$ populated in the present experiment), it can be 


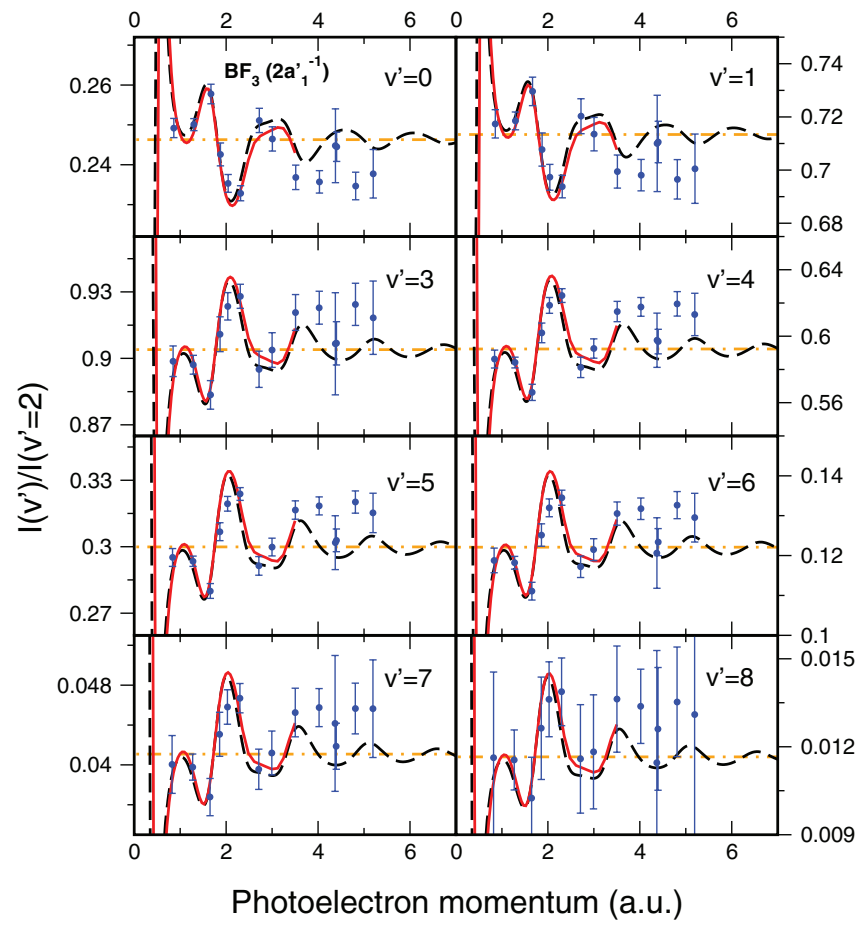

FIG. 3. Ratios between vibrationally resolved photoionization cross sections in $\mathrm{B} 1 s$ photoionization of $\mathrm{BF}_{3}$ in the symmetric stretching mode. The photoelectron momentum is defined with respect to the ground vibronic state of $\mathrm{BF}_{3}^{+}$. All the ratios are referred to the $v=2$ cross section. Circles with error bars: experimental data including statistical errors. Red full lines: results of the TDDFT calculations. Black dashed lines: results from the static-exchange DFT calculations. Horizontal dashed-dotted lines: ratios predicted by the FC approximation.

easily shown that the vibrationally resolved photoionization cross section for the totally symmetric mode can be written as

$$
\sigma_{v}(E) \simeq \mathrm{FC}_{v}\left[\sigma_{0}(E)+v \sigma_{1}(E)\right]
$$

where $\mathrm{FC}_{v}$ is the Franck-Condon factor $\mathrm{FC}_{v}=\left|\left\langle\chi_{0} \mid \chi_{v}\right\rangle\right|^{2}$ with $\chi_{0}$ and $\chi_{v}$ the initial and final vibrational wave functions in the symmetric stretching mode, $\sigma_{0}$ is the photoionization cross section of the rigid molecule at its equilibrium geometry, and $\sigma_{1}$ is the first order correction due to the fact that the molecule is flexible. The total cross section is obviously given by

$$
\sigma_{\mathrm{Tot}} \simeq \sigma_{0}+\bar{v} \sigma_{1}, \quad \bar{v}=\sum_{v} \mathrm{FC}_{v} v .
$$

The quantity $(v-\bar{v}) \sigma_{1}$, which does not appear explicitly in the above expressions, describes the fluctuations of the barycenter of the photoelectron-peaks envelope with respect to the $\mathrm{FC}$ value. Hence, it is convenient to introduce a $\mathcal{G}$ factor defined as

$$
\mathcal{G}=\sigma_{1} / \sigma_{\mathrm{Tot}},
$$

with which the vibrationally resolved cross section and the corresponding $v$-ratio, $\mathcal{R}$, take on the form

$$
\begin{gathered}
\sigma_{v}(E) \simeq \sigma_{\mathrm{Tot}}(E) \mathrm{FC}_{v}[1+(v-\bar{v}) \mathcal{G}(E)], \\
\mathcal{R}_{v, v_{0}}(E) \simeq\left(\mathrm{FC}_{v} / \mathrm{FC}_{v_{0}}\right)\left[1+\left(v-v_{0}\right) \mathcal{G}(E)\right] .
\end{gathered}
$$

From Eq. (5), it is apparent that the oscillatory behavior observed in the $v$-ratios is entirely contained in the universal function $\mathcal{G}(E)$, which in addition does not include the fast decrease of the total cross section.

By using the measured and DFT-calculated $v$-ratios in Eq. (5), we can extract experimental and theoretical values of $\mathcal{G}$. They are shown in Fig. 4. As for the $v$-ratios shown in Fig. 3, the agreement between theory and experiment is good for the three molecules. It is important to note that, since the $\mathcal{G}$ factor contains information from all the measured $v$ ratios, statistical errors are substantially reduced with respect to those of the individual $v$-ratios, especially for $\mathrm{BF}_{3}$. It can be seen that oscillations are quite apparent and have the characteristic momentum periodicity $\Delta k=\pi / R_{0}^{\mathrm{AB}_{\mathrm{n}}}$, where $R_{0}^{\mathrm{AB}_{\mathrm{n}}}$ is the bond distance of the $\mathrm{AB}_{n}$ molecule at equilibrium.

To prove without ambiguity that the observed oscillations are entirely due to diffraction of the ejected photoelectron, we have also used a simple analytical model to evaluate $\mathcal{G}(E){ }^{48}$ The model is based on a one-particle description of $\mathrm{AB}_{n}$ photoionization in which the interaction between photoelectron and ion is approximated by a sum of atomic Yukawa potentials, the initial electronic wave function is represented by a purely hydrogenic $1 s$ orbital, and the final wave function $\left|\psi_{\vec{k}}^{-}\right\rangle$ is described within the first Born approximation:

$$
\left|\psi_{\vec{k}}^{-}\right\rangle=|\vec{k}\rangle+G_{0}^{-}(E) V|\vec{k}\rangle
$$

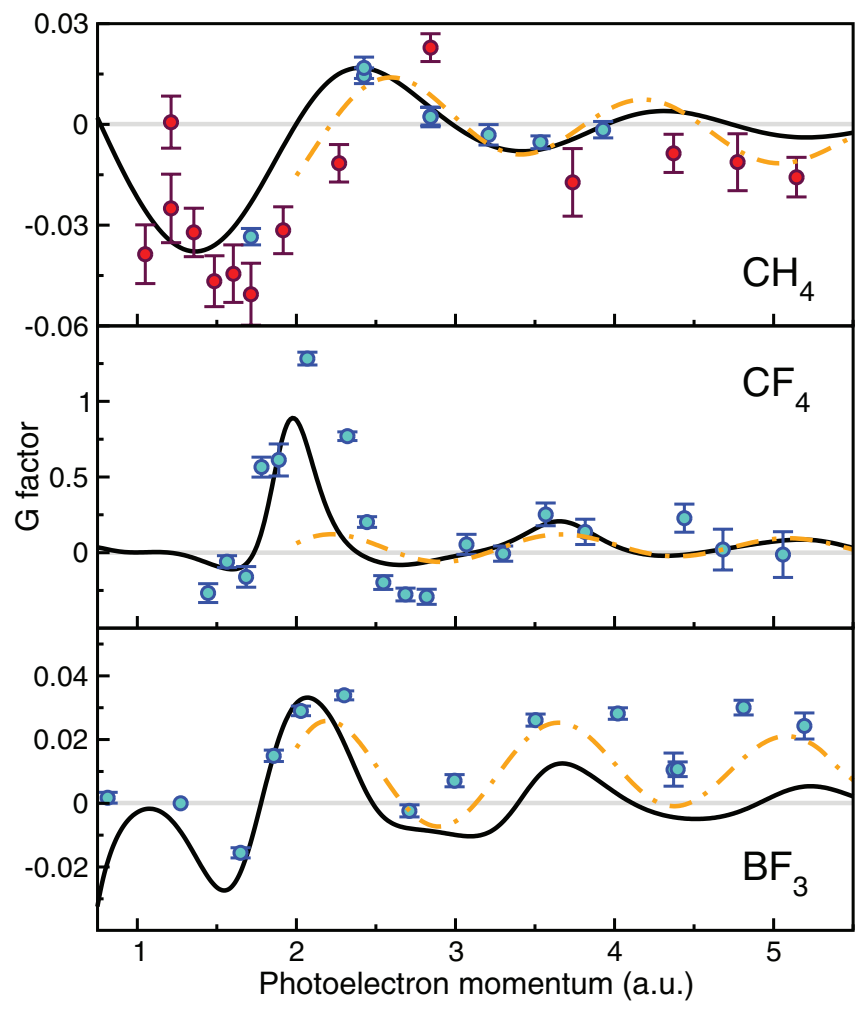

FIG. 4. Generalized $v$-ratio ( $\mathcal{G}$ factor) for $\mathrm{CH}_{4}, \mathrm{CF}_{4}$, and $\mathrm{BF}_{3}$ as a function of photoelectron momentum. Circles with error bars: experimental results including statistical errors. Black full lines: results from the static-exchange DFT calculations. Orange dashed-dotted lines: results of the simple analytical model explained in the text. For $\mathrm{CH}_{4}$, the $\mathcal{G}$ factor has been evaluated from the experimental and theoretical data of Ref. 26 and the experimental data of Ref. 47 (red circles). 
where $|\vec{k}\rangle$ is a plane wave, $G_{0}^{-}$is the corresponding Green's function, and $V$ is the electron-ion potential. Under these assumptions, all structural information is contained in the scattering term $G_{0}^{-}(E) V|\vec{k}\rangle$. Neglecting non-totally symmetric modes and averaging over all molecular orientations, Eq. (3) leads to ${ }^{48}$

$$
\begin{aligned}
\mathcal{G}\left(E, \tilde{R}_{0}, Z_{B}, \phi\right) \\
=\frac{2 Z_{B}}{\omega \Delta R M_{B}}\left[2 \cos \phi \int_{0}^{\infty} d q \frac{q^{2} J(q, k)}{q^{2}+1} j_{1}\left(q \tilde{R}_{0}\right)\right. \\
\left.\quad-\pi \sin \phi 1 s(k) \int_{-1}^{1} d x \frac{k^{3} x \sqrt{2-2 x}}{2 k^{2}(1-x)+1} j_{1}\left(\sqrt{2-2 x} k \tilde{R}_{0}\right)\right] \\
\quad \times\left[\pi k 1 s(k)-4 Z_{\mathrm{A}} \mathcal{P} \int_{0}^{\infty} d p \frac{1 s(p) p^{3}}{k^{2}-p^{2}} \int_{-1}^{1} \frac{x d x}{p^{2}+k^{2}-2 p k x+1}\right]^{-1},
\end{aligned}
$$

where $E=k^{2} / 2, \tilde{R}_{0}=\left(R_{0}^{A B_{n}}+R_{0}^{A B_{n}^{+}}\right) / 2$ is the average equilibrium distance, $\Delta R=R_{0}^{A B_{n}}-R_{0}^{A B_{n}^{+}}=\sqrt{2 \bar{v} / n \omega M_{\mathrm{B}}}$ is the difference in bond length between the neutral molecule and the cation, $\phi$ is the intramolecular scattering phase, $1 s(k)$ is the initial $1 s$ orbital in momentum representation, $j_{1}$ is the spherical Bessel function of order one, $Z_{B}$ and $M_{B}$ are, respectively, the effective charge and the mass of atom $\mathrm{B}$, and

$$
J(q, k)=\int_{-1}^{1} d x \frac{k+q x}{q+2 k x} 1 s\left(\sqrt{k^{2}+q^{2}+2 k q x}\right) .
$$

Thus, Eq. (7) may be employed to determine $\tilde{R}_{0}$ (and also $Z_{B}$ and $\phi$ ) by a least-squares fit to the experimental data. The resulting values for the $\mathrm{C}-\mathrm{H}$ and $\mathrm{B}-\mathrm{F}$ bond lengths of the neutral (charged) $\mathrm{CH}_{4}$ and $\mathrm{BF}_{3}$ molecules are 1.08 (1.03) and 1.20 (1.13) A, respectively, in reasonable agreement with the experimental values 1.09 (1.04) and $1.31(1.25) \AA .^{46,49} \mathrm{~A}$ similar fit is not possible for $\mathrm{CF}_{4}$ due to the failure of the first Born approximation up to relatively high $k$. It is worth noticing that a fit of Eq. (7) to the static-exchange DFT data, which contain hundreds of points, leads to an even better agreement with the experimental bond length values, including $\mathrm{CF}_{4}$. These results confirm the validity of the assumptions leading to the $\mathcal{G}$ function and prove that the relevant structural information for the neutral molecule and the cation is contained in that function. Still, there is room to improve the accuracy of the fitted bond lengths, e.g., by removing some of the approximations used to derive Eq. (7).

\section{CONCLUSION}

We have performed high resolution experiments and static-exchange density functional theory and time-dependent density functional theory calculations to determine $\mathrm{K}$-shell vibrationally resolved photoionization spectra of $\mathrm{CF}_{4}, \mathrm{BF}_{3}$, and $\mathrm{CH}_{4}$. The results show that the ratios between vibrationally resolved cross sections, the so-called $v$-ratios, exhibit pronounced oscillations as a function of photoelectron energy. These oscillations are the fingerprint of electron diffraction by the peripheral atomic centers. The observation of this phenomenon has been possible due to (i) the significant improvement in experimental resolution and statistics with respect to earlier experiments, (ii) the measurements and calculations performed for three different molecular systems containing quite different atomic species, and (iii) a new theoretical framework that allows one to analyze the observed diffraction patterns in a unified way irrespective of the molecule under consideration. The latter framework is based on a simple first-order treatment of atomic displacements and has been used to determine the bond lengths of neutral $\mathrm{BF}_{3}$ and $\mathrm{CH}_{4}$, as well as of their corresponding cations with a reasonable accuracy. This is a surplus of photoelectron spectroscopy with respect to more conventional spectroscopic techniques, which usually can only provide structural information of neutral molecular species.

Vibrationally resolved photoelectron spectroscopy allows detecting intramolecular electron diffraction in an elegant and consistent way, because (i) the problem of the rapid decrease of the photoionization probability with photon energy can be avoided by monitoring the ratios between vibrationally resolved cross sections (as opposed to the unresolved total cross section), and (ii) the effect of diffraction interferences manifest differently in different final vibrational states. High-resolution third-generation synchrotron facilities have made it possible to resolve the photoelectron peaks corresponding to different vibrational states of the parent ion, thus demonstrating unambiguously and conclusively that the nuclear response to intramolecular electron diffraction is observable and therefore could be used to obtain structural information of neutral and ionic symmetric polyatomic molecules.

\section{ACKNOWLEDGMENTS}

We thank Mare Nostrum BSC, Cineca, and CCCUAM for allocation of computer time. Work supported by the Advanced Grant of the European Research Council XCHEM 290853, the MICINN Project Nos. FIS2010-15127, ACI2008-0777, and CSD 2007-00010 (Spain), the ERAChemistry Project No. PIM2010EEC-00751, the European GrantNos. MC-ITN CORINF and MC-RG ATTOTREND, the European COST Action CM0702, the MC-IEF CHICROCSX under Grant Agreement No. 252781 (OT) and I3 program, the MEXT and JSPS agencies (Japan), and the Academy of Finland. Data collection was performed at the PLEIADES beamline at SOLEIL Synchrotron, France (Proposal No. 20110781). We are grateful to T. D. Thomas for stimulating discussions, to the SOLEIL staff for smoothly running the facilities and to E. Robert for technical assistance.

${ }^{1}$ D. E. Sayers, E. A. Sterntf, and F. W. Lytle, Phys. Rev. Lett. 27, 1204 (1971).

${ }^{2}$ R. F. Pettifer, O. Mathon, S. Pascarelli, M. D. Cooke, and M. R. J. Gibbs, Nature (London) 435, 78 (2005).

${ }^{3}$ S. A. Chambers, Adv. Phys. 40, 357 (1991).

${ }^{4}$ P. Hofmann, K. M. Schindler, S. Bao, A. M. Bradshaw, and D. P. Woodruff, Nature (London) 368, 131 (1994).

${ }^{5}$ P. Glatzel and U. Bergmann, Coord. Chem. Rev. 249, 65 (2005).

${ }^{6}$ C. Bressler and M. Chergui, Chem. Rev. 104, 1781 (2004).

${ }^{7}$ D. Woodruff, Surf. Sci. Rep. 62, 1 (2007).

${ }^{8}$ A. Arcovito, M. Benfatto, M. Cianci, S. S. Hasnain, K. Nienhaus, G. U. Nienhaus, C. Savino, R. W. Strange, B. Vallone, and S. D. Longa, Proc. Natl. Acad. Sci. U.S.A. 104, 6211 (2007). 
${ }^{9}$ M. Haumann, A. Grundmeier, I. Zaharieva, and H. Dau, Proc. Natl. Acad. Sci. U.S.A. 105, 17384 (2008).

${ }^{10}$ N. Pangher, H. M. Köppe, J. Feldhaus, and J. Haase, Phys. Rev. Lett. 71, 4365 (1993).

${ }^{11}$ R. Prešeren, A. Kodre, I. Arčon, and M. Borowski, J. Synchrotron Radiat. 8, 279 (2001).

${ }^{12}$ J. Söderström, N. Mårtensson, O. Travnikova, M. Patanen, C. Miron, L. J. Saethre, K. J. Børve, J. J. Rehr, J. J. Kas, F. D. Vila, T. D. Thomas, and S. Svensson, Phys. Rev. Lett. 108, 193005 (2012).

${ }^{13}$ F. Krasniqi, B. Najjari, L. Strüder, D. Rolles, A. Voitkiv, and J. Ullrich, Phys. Rev. A 81, 033411 (2010).

${ }^{14}$ M. Meckel, D. Comtois, D. Zeidler, A. Staudte, D. Pavicic, H. C. Bandulet, H. Pépin, J. C. Kieffer, R. Dörner, D. M. Villeneuve, and P. B. Corkum, Science 320, 1478 (2008).

${ }^{15}$ M. Okunishi, H. Niikura, R. Lucchese, T. Morishita, and K. Ueda, Phys. Rev. Lett. 106, 3 (2011).

${ }^{16}$ C. I. Blaga, J. Xu, A. D. DiChiara, E. Sistrunk, K. Zhang, P. Agostini, T. A. Miller, L. F. DiMauro, and C. D. Lin, Nature (London) 483, 194 (2012).

${ }^{17}$ H. Fukuzawa, X.-J. Liu, T. Teranishi, K. Sakai, G. Prümper, K. Ueda, Y. Morishita, N. Saito, M. Stener, and P. Decleva, Chem. Phys. Lett. 451, 182 (2008).

${ }^{18}$ J. Adachi, M. Kazama, T. Teramoto, N. Miyauchi, T. Mizuno, M. Yamazaki, T. Fujikawa, and A. Yagishita, J. Phys. B 45, 194007 (2012).

${ }^{19}$ J. B. Williams, C. S. Trevisan, M. S. Schöffler, T. Jahnke, I. Bocharova, H. Kim, B. Ulrich, R. Wallauer, F. Sturm, T. N. Rescigno, A. Belkacem, R. Dörner, T. Weber, C. W. McCurdy, and A. L. Landers, Phys. Rev. Lett. 108, 233002 (2012).

${ }^{20}$ J. B. Williams, C. S. Trevisan, M. S. Schöffler, T. Jahnke, I. Bocharova, H. Kim, B. Ulrich, R. Wallauer, F. Sturm, T. N. Rescigno, A. Belkacem, R. Dörner, T. Weber, C. W. McCurdy, and A. L. Landers, J. Phys. B 45, 194003 (2012).

${ }^{21}$ R. Dörner, V. Mergel, O. Jagutzki, L. Spielberger, J. Ullrich, R. Moshammer, and H. Schmidt-Böcking, Phys. Rep. 330, 95 (2000).

${ }^{22}$ C. Miron and P. Morin, Nucl. Instrum. Methods Phys. Res. A 601, 66 (2009)

${ }^{23}$ S. E. Canton, E. Plésiat, J. D. Bozek, B. S. Rude, P. Decleva, and F. Martín, Proc. Natl. Acad. Sci. U.S.A. 108, 7302 (2011).

${ }^{24}$ X.-J. Liu, N. A. Cherepkov, S. K. Semenov, V. Kimberg, F. Gel'mukhanov, G. Prümper, T. Lischke, T. Tanaka, M. Hoshino, H. Tanaka, and K. Ueda, J. Phys. B 39, 4801 (2006).

${ }^{25}$ L. Argenti, T. D. Thomas, E. Plésiat, X.-J. Liu, C. Miron, T. Lischke, G. Prümper, K. Sakai, T. Ouchi, R. Püttner, V. Sekushin, T. Tanaka, M. Hoshino, H. Tanaka, P. Decleva, K. Ueda, and F. Martín, New J. Phys. 14, 033012 (2012).

${ }^{26}$ E. Plésiat, L. Argenti, E. Kukk, C. Miron, K. Ueda, P. Decleva, and F. Martín, Phys. Rev. A 85, 023409 (2012).

${ }^{27}$ I. Hargittai and M. Hargittai, Stereochemical Applications of Gas-Phase Electron Diffraction (VCH, New York, 1988), Vols. 1 and 2.
${ }^{28}$ S. A. Hayes, A. Stuart, R. J. Berger, B. Neumann, N. W. Mitzel, J. Bader, and B. Hoge, Dalton Trans. 39, 5630 (2010).

${ }^{29}$ C. E. Knapp, D. A. Wann, A. Bil, J. T. Schirlin, H. E. Robertson, P. F. McMillan, D. W. H. Rankin, and C. J. Carmalt, Inorg. Chem. 51, 3324 (2012).

${ }^{30}$ C. J. Hensley, J. Yang, and M. Centurion, Phys. Rev. Lett. 109, 133202 (2012).

${ }^{31}$ E. Plésiat, P. Decleva, and F. Martín, Phys. Chem. Chem. Phys. 14, 10853 (2012).

${ }^{32}$ C. Miron, see www.synchrotron-soleil.fr/Recherche/LignesLumiere/ PLEIADES for complete information about the PLEIADES beamline at SOLEIL.

${ }^{33}$ C. Miron, C. Nicolas, O. Travnikova, P. Morin, Y. Sun, F. Gel'mukhanov, N. Kosugi, and V. Kimberg, Nature Phys. 8, 135 (2012).

${ }^{34}$ A. Lindblad, V. Kimberg, J. Söderström, C. Nicolas, O. Travnikova, N. Kosugi, F. Gel'mukhanov, and C. Miron, New J. Phys. 14, 113018 (2012).

${ }^{35}$ E. Kukk, Spectral Analysis by Curve Fitting Macro Package SPANCF 2000; See www.physics.utu.fi/en/department/materials_research/ materials_science/Fitting.html for details of the lcode used to carry out the least-squares fit of the experimental data.

${ }^{36}$ P. van der Straten, R. Morgenstern, and A. Niehaus, Z. Phys. D: At., Mol. Clusters 8, 35 (1988).

${ }^{37}$ Y.-P. Sun, C.-K. Wang, and F. Gelmukhanov, Phys. Rev. A 82, 052506 (2010).

${ }^{38}$ T. D. Thomas, E. Kukk, K. Ueda, T. Ouchi, K. Sakai, T. X. Carroll, C. Nicolas, O. Travnikova, and C. Miron, Phys. Rev. Lett. 106, 193009 (2011).

${ }^{39}$ B. Paripás, G. Vitéz, G. Víkor, K. Tökési, A. Caló, R. Sankari, M. Huttula, S. Aksela, and H. Aksela, J. Phys. B 37, 4507 (2004).

${ }^{40}$ T. D. Thomas, E. Kukk, R. Sankari, H. Fukuzawa, G. Prümper, K. Ueda, R. Püttner, J. R. Harries, Y. Tamenori, T. Tanaka, M. Hoshino, and H. Tanaka, J. Chem. Phys. 128, 144311 (2008).

${ }^{41}$ M. Stener, G. De Alti, and P. Decleva, Theor. Chem. Acc. 101, 247 (1999).

${ }^{42}$ M. Stener and P. Decleva, J. Chem. Phys. 112, 10871 (2000).

${ }^{43}$ M. Stener, G. Fronzoni, and P. Decleva, Chem. Phys. Lett. 351, 469 (2002).

${ }^{44}$ M. Stener, D. Toffoli, G. Fronzoni, and P. Decleva, J. Chem. Phys. 124, 114306 (2006).

${ }^{45}$ H. Bachau, E. Cormier, P. Decleva, J. E. Hansen, and F. Martín, Rep. Prog. Phys. 64, 1815 (2001).

${ }^{46}$ T. D. Thomas, R. Püttner, H. Fukuzawa, G. Prümper, K. Ueda, E. Kukk, R. Sankari, J. Harries, Y. Tamenori, T. Tanaka, M. Hoshino, and H. Tanaka, J. Chem. Phys. 127, 244309 (2007).

${ }^{47}$ E. Kukk, K. Ueda, U. Hergenhahn, X.-J. Liu, G. Prümper, H. Yoshida, Y. Tamenori, C. Makochekanwa, T. Tanaka, M. Kitajima, and H. Tanaka, Phys. Rev. Lett. 95, 133001 (2005).

${ }^{48}$ L. Argenti, E. Plésiat, P. Decleva, and F. Martín (unpublished).

${ }^{49}$ T. X. Carroll, N. Berrah, J. Bozek, J. Hahne, E. Kukk, L. J. Sæthre, and T. D. Thomas, Phys. Rev. A 59, 3386 (1999). 